\title{
Splenic Abscess and Management Options: Report of Four Cases
}

\author{
Mohammed Al-Jabali ${ }^{\mathrm{a}, \mathrm{b}}$, Bandar Idrees Alia , Abdallah Al-Zahrani ${ }^{\mathrm{a}}$, \\ Tagreed Al-Ajai ${ }^{\mathrm{a}}$
}

\begin{abstract}
Abscess of the spleen is a rather clinical rarity with reported mortality rate up to $47 \%$. The timely and widespread use of imaging methods facilitates early diagnosis and guides treatment, thus improving the prognosis. Most of patients were with recognized risk factors including conditions that compromise the immune system, trauma and intravenous drug abuse in addicts. The surgical treatment by splenectomy is usually the first choice of treatment but percutaneous drainage is the valid option. Splenic abscess is an unusual and potentially lifethreatening disease with a diagnostic challenge due to the non-specific clinical picture and diagnosis is confirmed based mostly on imaging studies. Splenic abscesses are very rarely encountered in surgical practice with a reported high mortality rate in neglected and untreated cases. Percutaneous drainage is a valid option and safe procedure for patients with splenic abscess.
\end{abstract}

Keywords: Splenic abscess; Management; Percutaneous drainage

\section{Introduction}

Abscess of the spleen is a rare discovery. Although it may have various causes, it is most usually associated with trauma and infections of the spleen. The latter are more common in the presence of a different primary site of infection, especially endocarditis or in cases of ischemic infarcts that are secondarily infected. Moreover, immunosuppression is a major risk factor [1-3]. Clinical examination usually reveals a combination of fever, left upper quadrant abdominal pain and vomiting [1-6]. Laboratory findings are not constant. Imaging is a necessary tool for establishing the diagnosis, with a choice between ultrasound (US) and computed tomography (CT). Treatment in-

Manuscript accepted for publication September 01, 2016

${ }^{a}$ Department of Surgery, Prince Sultan Military Medical City, Riyadh, Saudi Arabia

${ }^{b}$ Corresponding Author: Mohammed Al Jabali, Division of Upper GI \& Bariatric Surgery, Department of General Surgery, PSMMC, Riyadh, Saudi Arabia. Email:mfgalg@gmail.com

doi: http://dx.doi.org/10.14740/jmc2633w cludes conservative measures, percutaneous drainage and surgical intervention. Recently, abscess drainage is proved to be an efficient method in the treatment of splenic abscess $[4,5]$. Here, we present four cases of splenic abscess, with different approaches.

\section{Case Reports}

\section{Case 1}

A 75-year-old female patient, known to have diabetic mellitus, ischemic heart disease and atrial fibrillation, presented to emergency department with sudden, severe generalized abdominal pain and distension for 1 day. Patient gave a history of abdominal pain and ill-being for more than 3 weeks. Patient was found in septic shock with abdominal distention and generalized tenderness. Laboratory testing revealed a sign of leukocytosis, dehydration and metabolic acidosis. Blood cultured grew a Klebsiella pneumonia. Imaging tests including chest and abdomen X-rays were unremarkable followed by a CT scan of the abdomen which showed splenic collection with intra-abdominal free fluid (Fig. 1). Echocardiogram did not show any intra-cardiac vegetation.

Open splenectomy was done and patient had recovered well after a stormy 3-week intensive care unit (ICU) stay, due to septic shock and ARDS.

\section{Case 2}

Patient was an 80-year-old male known to have diabetes mellitus, hypertension and atrial fibrillation. Patient was hospitalized for 1 month due to ischemic stroke. During his hospital stay, he developed unexplained fever and bacteremia without documented infection source. Clinical examination reproduced localized mild tenderness over the left upper quadrant, with no other significant findings. Laboratory testing revealed a leukocytosis. Blood culture was growing a Gram-negative rode Klebsiella (multi-drug resistance (MDR)). Full septic workup included abdominal CT which showed a splenic abscess (Fig. 2).

Aspiration of the abscess was performed under US guidance drainage and the culture of aspirated fluid grew a Kleb- 


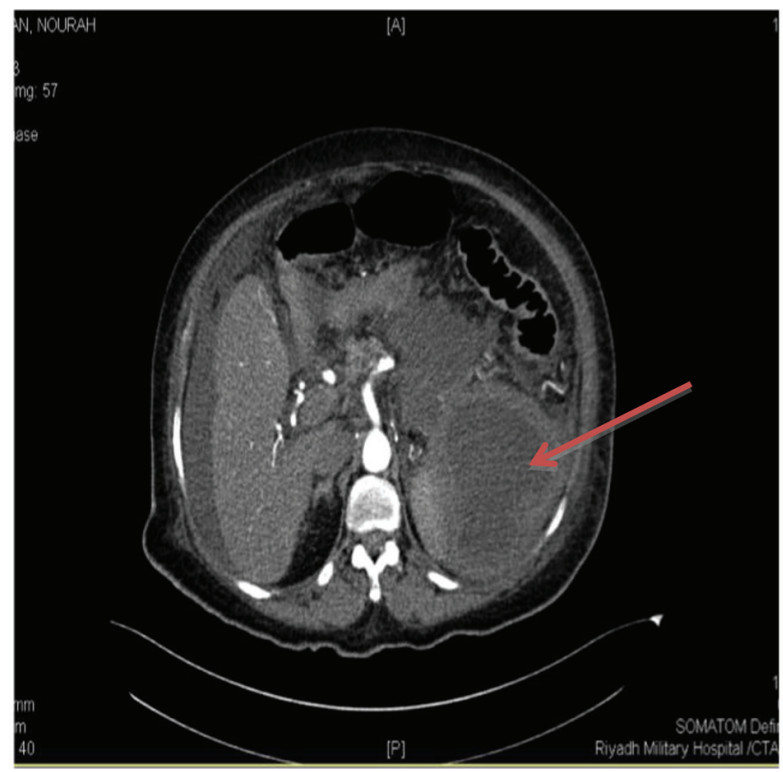

Figure 1. Abdominal CT scan of a 75-year-old female. The spleen contained a large abscess.

siella pneumonia multiple resistant strain. Patient recovered well with antibiotics therapy and serial US showed resolution of splenic abscess.

\section{Case 3}

A 70-year-old male patient with multiple co-morbidities was admitted to the hospital as a victim of RTA with tibia bone fracture treated with internal fixation. Ten days after his admission, he was transferred to the ICU with unexplained fever and decreased level of consciousness. Septic screen was done and it showed singes of acute infection. Blood culture grew a Gram-negative rode (Klebsilae pneumonia) and abdominal CT scan showed a huge splenic abscess (Fig. 3).

Patient was critically ill, but US-guided percutaneous drainage failed due to thick pus and debris. So patient was ex-

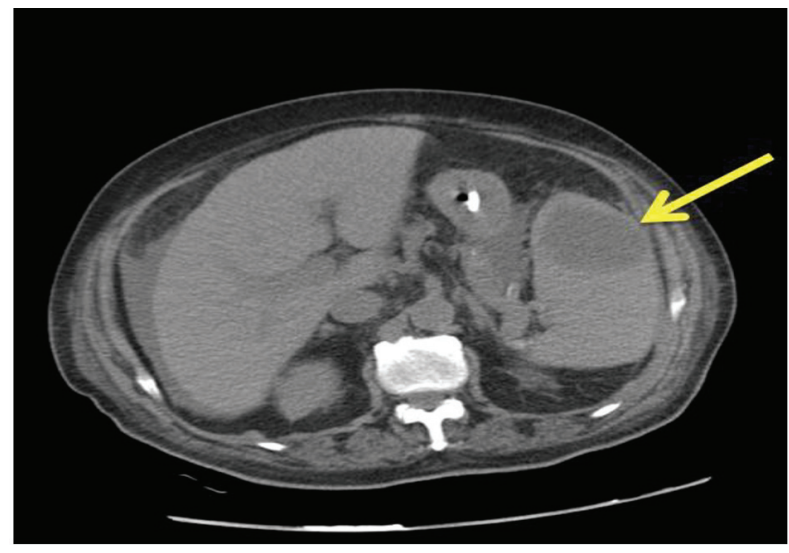

Figure 2. A CT scan of an 80-year-old man showing a large single abscess of $4 \times 4 \mathrm{~cm}$ in the upper pole of the spleen.

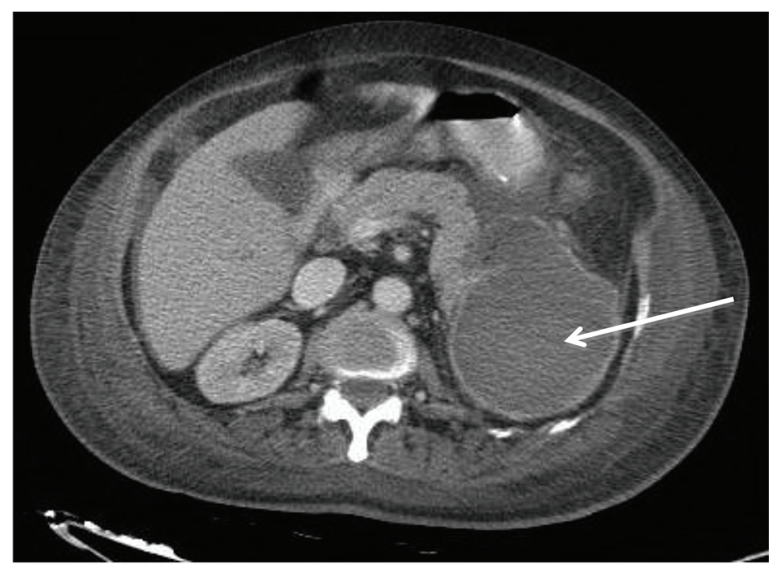

Figure 3. Abdominal CT of a 70-year-old male with large splenic abscess.

plored and splenectomy was done.

Patient had stormy post-operative course and had stool leak requiring a left colectomy and end colostomy and later patient died secondary to profound sepsis.

\section{Case 4}

Patient was a 35-year-old female with status post-laparoscopic sleeve gastrectomy. She presented to the emergency department 3 weeks after surgery with left upper quadrant abdominal pain and fever. Clinical examination revealed tenderness over the left upper quadrant with no other remarkable findings. CT scan was done to investigate for any intra-abdominal collection, and she was found to have a splenic abscess (Fig. 4a, b).

Fine-needle aspiration of the abscess under US guidance was carried out, along with intravenous antibiotics. The material aspirated was cultured and it grew a Gram-positive cocci (Streptococcus milleri) as well as on blood culture. The abscess was resolved on a serial sonography. The patient had an uneventful recovery.

\section{Discussion}

Splenic abscess is an uncommon entity. The incidence of splenic abscess in various autopsy series has been estimated to be between $0.2 \%$ and $0.7 \%[7,8]$. However, this uncommon disease is recently being reported more frequently. Two main contributing organisms are found to be responsible for forming splenic abscess: Klebsilae pneumonia and streptococcus.

Two main contributing factors to the apparent increase in the incidence of splenic abscess are advances in imaging studies and an increased number of immunocompromised, trauma and cancer patients $[7,9]$. Splenic abscesses may often be misdiagnosed, because the signs and symptoms are nonspecific; nevertheless, modern imaging has improved the process of their diagnosis. In this series, treatment was carried out by antibiotics and percutaneous drainage or splenectomy. The mortality rate appeared to be related to the patient's general 

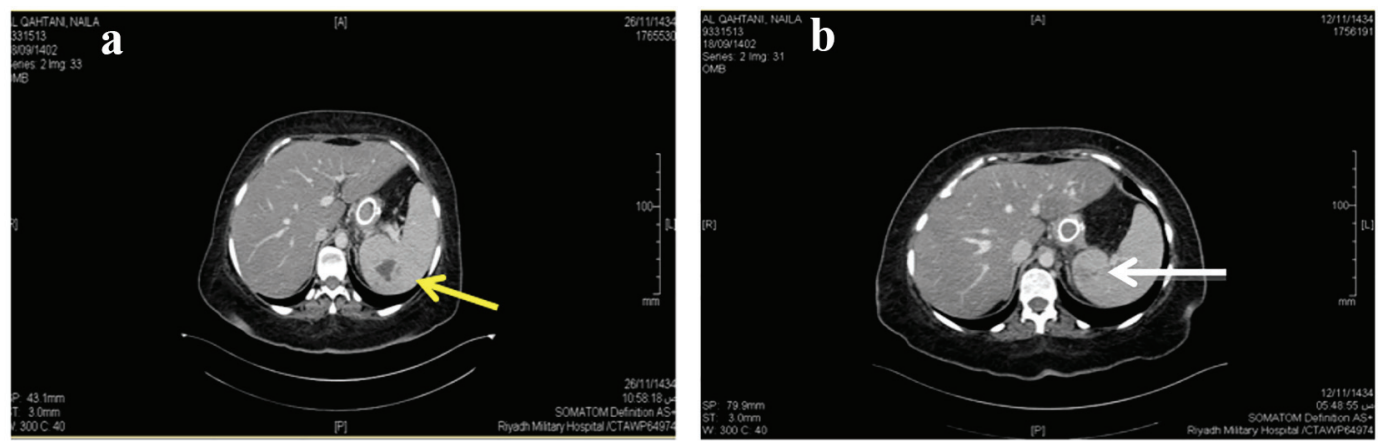

Figure 4. (a) Pre-drainage CT scan. (b) Post-drainage CT showing abscess in upper pole.

underlying condition. Our patients recovered from their splenic abscess regardless of the treatment modality. Thus, based on these observations, the prognosis for these patients cannot be accurately predicted by the intervention methods. Percutaneous treatment of splenic abscess is an effective alternative to surgery; furthermore, it offers the theoretical advantages of preserving immunologic function by avoiding splenectomy in young patients. The success rate of percutaneous drainage for splenic abscess has been reported between $67 \%$ and $100 \%$ [10].

Traditional treatment includes appropriate antimicrobial therapy with or without splenectomy. There are a number of studies in favor of spleen preservation and management using percutaneous drainage [10]. Percutaneous aspiration or drainage may be used as a bridge to surgery, allowing non-operative healing for splenic abscess patients who are at risk for surgery, and helps avoid the risk of a fulminant and potentially lifethreatening infection.

In conclusion, the best therapeutic approach for splenic abscess is still a matter of debate. We agree with the traditional treatment of antibiotics with or without splenectomy. However, based on our experience and the current literature, percutaneous aspiration of splenic abscess can be used as a bridge to surgery for those patients who are critically ill or who have several co-morbidities. Early diagnosis of splenic abscess requires a high degree of suspicion. Most of our patients presented with unexplained fever, abdominal pain and radiologic evidence of pathology in the left chest or abdomen, and these signs and symptoms should lead to suspicion of splenic abscess. Abdominal CT and ultrasonography were usually diagnostic. We suggest that treatment should be tailored and applied to splenic abscess patients on an individual basis.

\section{References}

1. Nelken N, Ignatius J, Skinner M, Christensen N. Changing clinical spectrum of splenic abscess. A multicenter study and review of the literature. Am J Surg. 1987;154(1):2734.

2. Pomerantz RA, Eckhauser FE, Thornton JW, Strodel WE, Knol JA, Zuidema GD. Covert splenic abscess: a continuing challenge. Am Surg. 1986;52(7):386-390.

3. Simson JN. Solitary abscess of the spleen. Br J Surg. 1980;67(2):106-110.

4. Goerg C, Schwerk WB, Goerg K. Splenic lesions: sonographic patterns, follow-up, differential diagnosis. Eur J Radiol. 1991;13(1):59-66.

5. Chou YH, Tiu CM, Chiou HJ, Hsu CC, Chiang JH, Yu C. Ultrasound-guided interventional procedures in splenic abscesses. Eur J Radiol. 1998;28(2):167-170.

6. Gadacz T, Way LW, Dunphy JE. Changing clinical spectrum of splenic abscess. Am J Surg. 1974;128(2):182187.

7. Teich S, Oliver GC, Canter JW. The early diagnosis of splenic abscess. Am Surg. 1986;52(6):303-307.

8. de Bree E, Tsiftsis D, Christodoulakis M, Harocopos G, Schoretsanitis G, Melissas J. Splenic abscess: a diagnostic and therapeutic challenge. Acta Chir Belg. 1998;98(5):199-202.

9. Tung CC, Chen FC, Lo CJ. Splenic abscess: an easily overlooked disease? Am Surg. 2006;72(4):322-325.

10. Thanos L, Dailiana T, Papaioannou G, Nikita A, Koutrouvelis $\mathrm{H}$, Kelekis DA. Percutaneous CT-guided drainage of splenic abscess. AJR Am J Roentgenol. 2002;179(3):629632. 